\title{
Vortex lattice in rotating neutron spin-triplet superfluid
}

\author{
Lev B. Leinson ${ }^{1 \star}$ \\ ${ }^{1}$ Pushkov Institute of Terrestrial Magnetism, Ionosphere and Radiowave Propagation of the Russian Academy of Science (IZMIRAN), \\ 108840 Troitsk, Moscow, Russia
}

Accepted XXX. Received YYY; in original form ZZZ

\section{INTRODUCTION}

When considering superfluid neutrons in rotating neutron stars, it is usually assumed that the superfluid neutron flux is everywhere irrotational. This approximation means that the velocity of superfluid neutrons $\mathbf{v}_{s}=\hbar \nabla \Phi / 2 M(M$ is the neutron mass) is determined exclusively by the phase gradient of the order parameter $\Psi=|\Psi| e^{i \Phi}$ and thus $\nabla \times \mathbf{v}_{s}=0$ in complete analogy with Bose-Einstein superfluids. In the equilibrium state, a superfluid liquid in a large rotating container makes a rigid body rotation as a whole, which is achieved by the presence of a lattice of quantized vortex lines. For a superfluid ${ }^{4} \mathrm{He}$ this was for the first time proved by Tkachenko (1965) who has shown that a triangular lattice of singular vortices is energetically the most favourable of all simple lattices.

The relations, derived for a superfluid ${ }^{4} \mathrm{He}$ in a rotating vessel are believed valid also for a neutron Fermi liquid in neutron stars (Richardson 1972; Muzikar et al. 1980; Sauls et al. 1982; Mendell 1991)). Obviously, this statement is true in the case of spin-singlet neutron pairing with the total spin of the Cooper pair $S=0$. In this case the condensate wave function has the form $\Psi_{S=0}=i \sigma_{2}|\Delta| e^{i \Phi}$, where $\boldsymbol{\sigma}=$ $\left\{\sigma_{1}, \sigma_{2}, \sigma_{3}\right\}$ are the Pauli spin matrices while the space part is a complex scalar function (for a recent review, for example, see (Haskell \& Sedrakian 2018; Sedrakian \& Clark 2019)). One again has $\mathbf{v}_{s} \propto \nabla \Phi$ and $\nabla \times \mathbf{v}_{s}=0$, similar to superfluid ${ }^{4} \mathrm{He}$ in the rotating bucket.

The spin-singlet neutron pairing is believed to take

^ E-mail: leinson@yandex.ru place in the outer part of the neutron star core and in the inner crust (Chamel \& Haensel 2008) while, in the inner core, the Cooper pairing of neutrons occurs ${ }^{1}$ with the orbital momentum $L=1$ and the total spin $S=1$. This significantly complicates the behavior of superfluid neutrons. By this reason, in many pulsar glitch models, the S-wave and $\mathrm{P}$-wave superfluids are treated as different entities (Ho et al. 2015; Montoli et al. 2020). Due to the strong spin-orbit interaction, a ${ }^{3} \mathrm{P}_{2}$ state with the total momentum $J=2$ is realized (Tamagaki 1970; Takatsuka 1972). The wave function of a pair with momenta $\mathbf{p}$ and $-\mathbf{p}$ can be written in the form (Mermin \& Stare 1973; Fujita et al. 1978; Muzikar et al. 1980; Volovik 1984) (summation over repeated indices implied):

$\Psi=i|\Delta| \sigma_{i} \sigma_{2} \mathrm{~A}_{i j} \hat{p}_{j}$,

where $\hat{\mathbf{p}}=\mathbf{p} / p$. The order parameter is a complex matrix $\mathrm{A}_{i j}$, which, due to the total momentum constraint $J=2$, must be symmetric and traceless: $\mathrm{A}_{i j}=\mathrm{A}_{j i}$ and $\mathrm{A}_{i i}=0$. This matrix is normally described in a local coordinate system in which the real part is diagonal and the off-diagonal part is purely imaginary (Richardson 1972; Sauls et al. 1982; Masuda \& Nitta 2016).

It is believed (Richardson 1972; Sauls et al. 1982; Masuda \& Nitta 2016) that the minimal energy corresponds to the order parameter of the form

$\mathrm{A}_{i j}=N e^{i \Phi}\left[\hat{u}_{i} \hat{u}_{j}+\lambda \hat{v}_{i} \hat{v}_{j}-(1+\lambda) \hat{w}_{i} \hat{w}_{j}\right]$,

1 We neglect a small contribution of tensor forces. 
associated with the triad of unit mutually orthogonal vectors $(\hat{u}, \hat{v}, \hat{w})$. Kinetic energy can be reduced by alignment $\hat{w}$ (eigenvector with the smallest eigenvalue) with $\mathbf{v}_{s}$ and by allowing the degeneracy parameter $\lambda$ to vary in the range $-1<\lambda<-1 / 2$. The planar distribution of $\hat{w}$ in this representation immediately leads to $\mathbf{v}_{s} \propto \nabla \Phi$ and $\nabla \times \mathbf{v}_{s}=0$, i.e. the minimal energy corresponds to a singular vertex with the superfluid velocity distribution similar to that shown in Fig. 1.

This result can be easily understood. Normally the distance $d$ between vortices in rapidly rotating neutron stars is much larger than the coherence length of superfluid neutrons $\xi$, and therefore the authors consider a single vortex (Richardson 1972; Sauls et al. 1982; Mendell 1991; Masuda \& Nitta 2016). It is well known that an isolated vortex in a rotating vessel of large radius can be stabilized only if its vorticity is concentrated in the central non-superfluid filament of size $\xi$ (a singular vortex). An isolated core-less vortex with a diffusely distributed vorticity are unstable.

However, core-less vortices are well known in the ${ }^{3} \mathrm{He}-$ A theory (Mermin \& Ho 1976; Chechetkin 1976; Volovik \& Kopnin 1977; Fujita et al. 1978; Volovik 1984). They have a vorticity diffusely distributed over the entire unit cell of the vortex lattice. For this reason, continuous vortices cannot be isolated and should be considered as a single periodic system.

The paper is organized as follows. In Sec. 2, we propose a reasonable trial state for a rotating ${ }^{3} \mathrm{P}_{2}$ superfluid system with coordinate-dependent directions of the triad of basis vectors written in terms of Euler angles. This type of order parameter allows one to consider both singular and non-singular vortices with distributed vorticity. The free energy of this vortex state in the Ginzburg-Landau regime is derived in the London limit. In Sec. 3, we discuss the mathematical functions used to describe the lattice of ordinary linear singular vortices in rotating superfluid ${ }^{4} \mathrm{He}$. Our analysis shows that the same approach can be used for a lattice of singular vortices in a rotating spin-triplet neutron superfluid, when the superfluid motion is determined by the phase gradient of the order parameter. In Sec. 4 we consider a more general form of the order parameter making use of the additional freedom provided by the coordinate dependence of orientation of the basis vectors triad in rotating spin-triplet superfluid. We construct the functions describing a lattice of non-singular vortices with a vorticity diffusely distributed over the entire elementary cell of the vortex lattice. Since it is difficult to solve the minimization problem, we construct some trial function which has the lattice symmetry. In Sec. 5 we use this trial function to calculate the free energy per area carrying a unit vorticity and compare it with the known energy of a lattice of linear singular vortices. Section 6 contains the summary of the obtained results. Possible consequences for the neutron star dynamics are discussed.

\section{FREE ENERGY OF THE ROTATING ${ }^{3} \mathbf{P}_{2}$ SUPERFLUID}

The diffusely distributed vorticity in a periodic system should be summed over the entire lattice into solid-state rotation, which is possible only if $\nabla \times \mathbf{v}_{s} \neq 0$. For the ${ }^{3} \mathrm{P}_{2}$ superfluid this can be the case due to additional degrees of freedom: coordinate-dependent directions of the triad of basis vectors. Indeed, if the condensate wave function is of the form of Eq. (1) with the matrix $A_{i j}$ depending on the centre of mass coordinate $\mathbf{r}=\left(\mathbf{r}_{1}+\mathbf{r}_{2}\right) / 2$ of a Cooper pair:

$\mathrm{A}_{i j}(\mathbf{r})=\frac{1}{2} e^{i \Phi(\mathbf{r})}\left(u_{1 i}+i u_{2 i}\right)\left(u_{1 j}+i u_{2 j}\right)$,

where $\mathbf{u}_{1}(\mathbf{r})$ and $\mathbf{u}_{2}(\mathbf{r})$ are real unit orthogonal vectors.

The normalized symmetric matrix $\mathrm{A}_{i j}$, as given by Eq. (2) is unitary, $A_{i j}^{*} A_{i j}=1$. Together with Eq. (1) it can be readily recognized as the wave function of the Cooper pairs in the ${ }^{3} \mathrm{P}_{2}$ state with $M_{J}=2$. The vector product $\mathbf{l}=\mathbf{u}_{1} \times \mathbf{u}_{2}$ indicates the direction of the quantization axis of the total angular momentum of the pair.

The current density at zero temperature is

$\mathbf{j}=\frac{\hbar}{4 i M} \sum_{\text {spin }}\left(\Psi^{\dagger} \nabla \Psi-\Psi \nabla \Psi^{\dagger}\right)=\rho_{s} \mathbf{v}_{s}$

From Eqs. (1) and (2) we find the invariant defined superfluid velocity (Volovik 1984):

$\mathbf{v}_{s}=\frac{\hbar}{2 M}\left(\nabla \Phi+2 u_{1 i} \boldsymbol{\nabla} u_{2 i}\right)$.

Summation over repeated indices $i, j=1,2,3$ is understood everywhere.

The triad $\left(\mathbf{u}_{1}, \mathbf{u}_{2}, \mathbf{l}\right)$ at any point can be specified by the Euler angles $(\Phi, \beta, S)$ which bring the standard Cartesian frame $(\mathbf{x}, \mathbf{y}, \mathbf{z})$ to the triad. In terms of these angles we have the matrix (2) with

$\mathbf{u}_{1}=(\cos S \cos \beta,-\sin S \cos \beta, \sin \beta)$,

$\mathbf{u}_{2}=(\sin S, \cos S, 0)$

and

$\mathbf{l}=(-\cos S \sin \beta, \sin S \sin \beta, \cos \beta)$.

Note that the order parameter (2) does not change upon simultaneous the gradient transformation and rotation of the spin-orbital space around the axis $\mathbf{l}$

$$
\begin{aligned}
\Phi & \rightarrow \Phi+\alpha \\
\mathbf{u}_{1}+i \mathbf{u}_{2} & \rightarrow \mathrm{R}\left(\frac{\alpha}{2} \mathbf{l}\right)\left(\mathbf{u}_{1}+i \mathbf{u}_{2}\right) .
\end{aligned}
$$

Therefore, instead of three variables (three angles specifying the orientation of the triplet of unit vectors $\mathbf{u}_{1}, \mathbf{u}_{2}, \mathbf{l}$ ), we introduced four, artificially separating the phase variable $\Phi$. In this case, we will assume that $\Phi$ changes only under the gradient transformation, and the unit vectors $\mathbf{u}_{1}, \mathbf{u}_{2}, \mathbf{l}$ only change under rotation. Thus $\mathbf{u}_{1}$ and $\mathbf{u}_{2}$ included in (4) are invariant under the gradient transformation.

The vorticity of superflow, $\nabla \times \mathbf{v}_{s}$ now depends on the $\mathbf{l}$ texture through the famous Mermin-Ho relation (Mermin \& Ho 1976):

$\boldsymbol{\nabla} \times \mathbf{v}_{s}=\frac{\hbar}{2 M} e_{i m n} l_{i}\left(\nabla l_{m} \times \nabla l_{n}\right)$.

Therefore, neutron superfluid with the order parameter (2) in the central core of a neutron stars is irrotational only when the right side of Eq. (9) equals zero; for example, in the case of planar field $\mathbf{l}$ (when the vector field $\mathbf{l}$ is concentrated in the plane of rotation).

To determine the equilibrium state, we have to find $\Phi$, $S$ and $\beta$, as well as lattice parameters that minimize free energy. Throughout this work we take the London limit, 
assuming spatial variations to be slow, in other words, we assume that non-uniform states are described by the phase $\Phi(r)$ and the field of the triad, while the condensation energy and hence $|\Delta|$ are kept constant. Since we take London limit, we have to consider only derivative terms in the free energy. The general form $F$ of this contribution to the free energy density, consistent with the spin-orbit rotation and uniform gauge invariance, near the critical temperature in the Ginzburg-Landau approximation can be written as

$F=\frac{\hbar^{2} \rho_{s}}{4 M^{2}}\left(\partial_{i} \overline{\mathrm{A}}_{k j} \partial_{i} \mathrm{~A}_{k j}+\partial_{i} \overline{\mathrm{A}}_{k j} \partial_{j} \mathrm{~A}_{k i}+\partial_{i} \bar{A}_{k i} \partial_{j} \mathrm{~A}_{k j}\right)$,

where the bar means a complex conjugate; $\rho_{s}$ denotes the superfluid density. In our approach this value is an external parameter, which can be determined, as a function of the density and temperature, from the standard GinzburgLandau theory.

Since we consider rotation of the superfluid it is convenient to use the frame of reference rotating with the container. Then the problem of minimizing the difference $F-\mathbf{L} \boldsymbol{\Omega}$ where $\mathbf{L}$ is the total angular momentum of the neutron liquid per unit length and $\boldsymbol{\Omega}$ is the angular velocity of the container rotation can be effectively reduced to a problem of the superfluid motion in the external vector potential $\mathbf{A}=M(\boldsymbol{\Omega} \times \mathbf{r})$. Therefore, accounting for the gauge invariance we obtain the relevant free energy by replacing $\partial_{i}$ by $\partial_{i}-2 i M(\boldsymbol{\Omega} \times \mathbf{r})_{i}$ in $(10)$.

The lattice period $d=2 a$ is uniquely determined by the rotation angular velocity. The vortex density $\eta_{v}=\omega / \pi$ in units of $1 / a^{2}$ and the angular velocity

$\boldsymbol{\omega}=\frac{2 M a^{2}}{\hbar} \boldsymbol{\Omega}$

are connected by Feynman's relation (Feynman 1965)

$\varkappa \eta_{v}=2 \omega$,

where $\varkappa$ is the vortex strength in units of $\pi \hbar / M$. We restrict ourselves to considering vortices with one quantum of circulation, $\varkappa=1$. In this case the phase $\Phi$ increases by $2 \pi$ when going around each point of the lattice.

It is convenient to use the dimensionless variable $\varrho=$ $\mathbf{r} / a=\{x, y, z\}$ meaning hereinafter $\boldsymbol{\nabla}=\left\{\partial_{x}, \partial_{y}, \partial_{z}\right\}$. Then from (10) we get

$\tilde{F}=\frac{\hbar^{2} \rho_{s}}{(2 M)^{2} a^{2}}\left(\bar{D}_{i} \overline{\mathrm{A}}_{k i} D_{j} \mathrm{~A}_{k j}+\bar{D}_{i} \overline{\mathrm{A}}_{k j} D_{i} \mathrm{~A}_{k j}+\bar{D}_{i} \overline{\mathrm{A}}_{k j} D_{j} \mathrm{~A}_{k i}\right)$,

where $\tilde{F}$ is the free energy density in the rotating frame, and

$\mathbf{D}=\nabla-i(\boldsymbol{\omega} \times \varrho), \quad \overline{\mathbf{D}}=\boldsymbol{\nabla}+i(\boldsymbol{\omega} \times \varrho)$.

In what follows we always use the rotating frame of reference unless stated otherwise. In the rotating frame of reference the superfluid moves relative a normal (non-superfluid) component with the velocity $\mathbf{v}_{s}-\mathbf{v}_{n}$, where $\mathbf{v}_{n}$ is the velocity of the normal matter co-rotating with the container. From Eq. (4) we obtain the dimensionless velocity in the rotating frame

$\mathbf{V} \equiv \frac{2 M a}{\hbar} \tilde{\mathbf{v}}_{s}=2 \cos \beta \nabla S+\nabla \Phi-\boldsymbol{\omega} \times \varrho$.
Making use of Eqs. (5)-(15) from Eq. (2) and (13) we get the derivative part of the free energy density

$\tilde{F}=\frac{\hbar^{2} \rho_{s}}{(2 M)^{2} a^{2}}\left\{\left(\mathbf{V} \mathbf{u}_{1}-\sin \beta \mathbf{l} \nabla S\right)^{2}+\left(\mathbf{V} \mathbf{u}_{2}-\mathbf{l} \nabla \beta\right)^{2}\right.$

$+\frac{1}{2}\left(\mathbf{V}-\mathbf{u}_{1} \times \nabla \beta\right)^{2}+\frac{1}{2}\left(\mathbf{V}+\sin \beta \mathbf{u}_{\mathbf{2}} \times \nabla S\right)^{2}$

$+\frac{3}{2} \sin ^{2} \beta\left(\mathbf{u}_{2} \boldsymbol{\nabla} S\right)^{2}+\frac{1}{2}\left(2+\sec ^{2} \beta\right)\left(\mathbf{u}_{1} \boldsymbol{\nabla} \beta\right)^{2}$

$\left.+\left(\mathbf{u}_{2} \boldsymbol{\nabla} \beta\right)^{2}+\frac{1}{4}(3+\cos (2 \beta)) \tan ^{2} \beta\left(\mathbf{u}_{1} \boldsymbol{\nabla} S\right)^{2}\right\}$.

\section{THE LATTICE OF ORDINARY VORTEX LINES}

Since the superfluid motion is actually two-dimensional it is convenient to define the complex coordinate $Z=x+i y$ in the plane at right angles to the vortices. We represent the velocity by a complex quantity $v(Z)$ whose magnitude and direction in the complex plane give the magnitude and direction of the fluid velocity at $Z$.

Let us consider first a lattice of ordinary vortex lines in a uniform texture, which corresponds to $\beta=$ const and $S=$ const. Without loss of generality, we can take $S=0$. If we assume that there exists a vortex at $Z=0$ then the coordinates of the other vortices will be $Z_{m n}=2 m+2 n i$, where $m$ and $n$ are arbitrary integers, 1 and $i$ are the halfperiods of the lattice (in units of $a$ ). Due to the irrotational nature of the flow in singular vortices, the complex velocity $v(Z)$ must be an analytic function (Milne-Thomson 1964), which has no other singularity in the finite plane, except for simple poles at the points $Z_{m n}$, where there are vortices, with identical residues. All simple lattices of this kind can undergo rigid rotation (Kiknadze et al. 1965). It is known that any functions of this kind whose poles form a regular lattice can be written in the form $\zeta_{0}(Z)=\zeta(Z)+f(Z)$, where $f(Z)$ is any entire function and $\zeta\left(Z ; g_{2}, g_{3}\right)$ is the zeta function of Weierstrass with invariants (the so-called modular forms) $g_{2}(1, i)$ and $g_{3}(1, i)$. For brevity, we mostly omit the arguments $g_{2}, g_{3}$ in the Weierstrass functions below.

Since in the general case it is difficult to solve the minimization problem, we consider some test structure in the form of a square lattice of ordinary singular vortices. In this case (Tkachenko 1965) one can put $f(Z)=0$ and the dimensionless superfluid velocity in the laboratory frame $v(Z) \equiv$ $(2 M a / \hbar) v_{s}(Z)$ can be written as $v(Z)=i \zeta\left(\bar{Z} ; g_{2}, g_{3}\right)$. In the rotating frame of reference, instead of Eq. (15), we get

$v_{r}(Z)=i \zeta\left(\bar{Z} ; g_{2}, g_{3}\right)-i \omega Z$,

where the second term represents the complex velocity $v_{n}$ of a non-superfluid component (a rigid rotation) and the bar means a complex conjugate $\bar{Z}=x-i y$.

To evaluate the phase function $\Phi$ (let us designate it $\Phi_{0}$ for the case of ordinary singular vortices), we consider the expression for complex potential (Gradshteyn \& Ryzhik 2007)

$\Psi(Z)=\int \zeta(Z) d Z=\ln \sigma\left(Z ; g_{2}, g_{3}\right)$,

where $\sigma\left(Z ; g_{2}, g_{3}\right)$ is the Weierstrass $\sigma$-function.

If we assume that $\Psi \equiv \varphi+i \psi$ is an analytic function of 


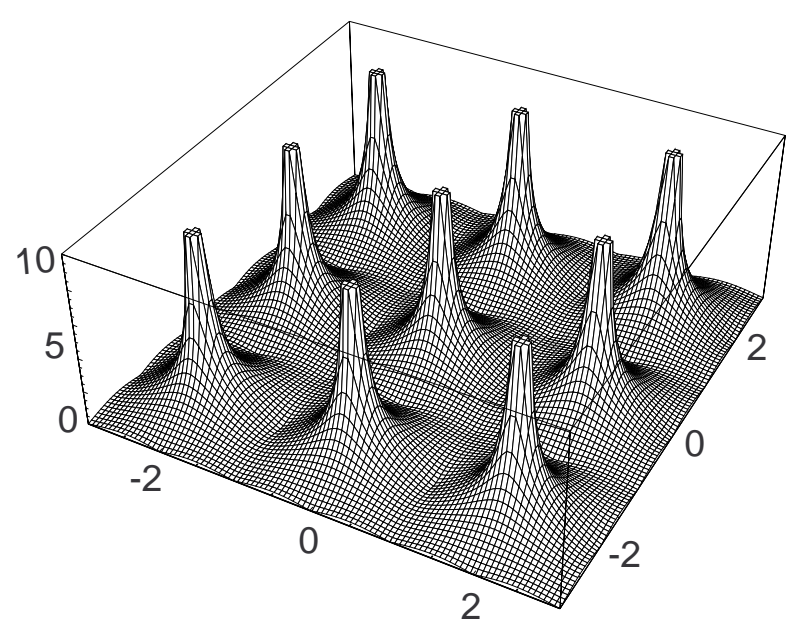

Figure 1. The lattice of ordinary linear singular vortices. The distribution of the absolute value of the superfluid velocity relative a normal component $\left|\mathbf{v}_{s}-\mathbf{v}_{n}\right|$ in the plane orthogonal to the rotation axis.

the variable $Z=x+i y$, then the real and imaginary parts of this function are the velocity potential and the stream function for some possible two-dimensional irrotational fluid motion. By virtue of the Cauchy-Riemann relations, any of these functions can be used to find the complex velocity. We make use of the expression

$\Phi_{0}=\Im \ln \sigma\left(Z ; g_{2}, g_{3}\right)$,

thus obtaining the complex velocity in the form

$i \zeta(x-i y)=-i \partial_{x} \Phi_{0}+\partial_{y} \Phi_{0}$,

The absolute value of $v_{r}(x, v)$ is shown in Figure 1.

This function has point singularity at each lattice site, where the entire vorticity is concentrated in the central nonsuperfluid filament of radius $\xi \ll a$.

\section{A LATTICE OF CORE-LESS VORTICES}

The lattice of linear singular vortices, discussed in the previous section, has been obtained in the case of $\beta=\pi / 2$ and $S=0$, when the superfluid motion is described by the phase $\Phi(\varrho)$ only.

We now remove the singular cores of the above vortex lattice by making use the remaining freedom in the order parameter - the field of the triad $\mathbf{u}_{1}, \mathbf{u}_{2}, \mathbf{l}$ with some trial functions $\beta(\varrho)$ and $S(\varrho)$. For this we notice that the matrix (2) can be recast as $\mathrm{A}_{i j}=u_{i} u_{j}$, where $\mathbf{u}$ is the vector of the form

$\mathbf{u}=\frac{1}{2} e^{i\left(\frac{1}{2} \Phi+S\right)}(1+\cos \beta) \mathbf{e}_{+}$

$+e^{i \frac{1}{2} \Phi} \sin \beta \mathbf{e}_{0}-\frac{1}{2} e^{i\left(\frac{1}{2} \Phi-S\right)}(1-\cos \beta) \mathbf{e}_{-}$

with

$\mathbf{e}_{ \pm}=\frac{1}{\sqrt{2}}(1, \pm i, 0), \quad \mathbf{e}_{0}=(0,0,1)$.

From this representation it is seen that where $\beta=\pi$ or
0 the singularity of the vortex type in the phase function $\frac{1}{2} \Phi+S$ or $\frac{1}{2} \Phi-S$ does not lead to divergent $\mathbf{V}$ [see Eq. (15)] provided $\frac{1}{2} \Phi \pm S$ is analytic. Given this, we choose two sets of lattice sites, where $\beta=0$ and $\pi$, that is, $\mathbf{l}$ is parallel and anti-parallel to $\boldsymbol{\omega}$, respectively: $\Phi_{0}(x, y)$ and $\Phi_{0}(x+1, y+1)$ and suppose

$\Phi(x, y)=\frac{1}{2}\left[\Phi_{0}(x+1, y+1)+\Phi_{0}(x, y)\right]$

and

$S(x, y)=\frac{1}{4}\left[\Phi_{0}(x+1, y+1)-\Phi_{0}(x, y)\right]$

We thus obtained the superfluid velocity (15) in the form of complex function

$\mathcal{V}=\frac{1}{2}(1+\cos \beta) i \zeta(x-i y+1-i)$

$+\frac{1}{2}(1-\cos \beta) i \zeta(x-i y)-i \omega(x+i y)$.

The Weierstrass zeta function has the following properties in a complex plane (Gradshteyn \& Ryzhik 2007):

$\zeta(Z+2 m)=\zeta(Z)+2 m \zeta(1)$,

$\zeta(Z+2 n i)=\zeta(Z)+2 n \zeta(i)$

$i \zeta(1)-\zeta(i)=i \pi / 2$.

If we take $\zeta(1)=\omega$ with $\omega=\pi / 4$, then from (28) it follows that $\zeta(i)=-i \omega$, so that the superfluid velocity becomes a periodic function and the free energy becomes the sum of that in one unit cell.

As was mentioned above, to determine the equilibrium state, we have to find the periodic function $\beta(x, y)$ that minimizes free energy (13). Since it is difficult to solve the minimization problem, we consider the trial function

$\beta=\frac{\pi}{2}\left[1-\frac{1}{2}(\cos \pi x+\cos \pi y)\right]$,

which has the lattice symmetry and is equal to 0 and $\pi$ at the pole points of the functions $i \zeta(x-i y)$ and $i \zeta(x-i y+1-i)$, respectively [see Eq.(25)].

The resulting velocity distribution is shown in Fig. 2, where the absolute value of the superfluid velocity in a rotating reference frame is depicted.

It can be seen that the superfluid velocity is a finite periodic function that has no singularity. To demonstrate the "topographic map" of the superfluid velocity amplitude, in Fig. 3, we present a contour plot of this function, which allows us to see the periodic lattice of continuous vortices with a vorticity distributed over the entire unit cell.

As can be seen, the modulus of the velocity $\left|\mathbf{v}_{s}-\mathbf{v}_{n}\right|$ is asymmetric. This fact has a simple explanation if we recall that the $\mathbf{l}$ axis indicates the direction of the quantization axis of the total angular momentum of the pair. Apart from the kinetic term, free energy (16) includes the distortion energy of the field $\mathbf{l}$ caused by twisting, splaying and bending, as in liquid crystals. In contrast to the case of ordinary linear singular vortices, for a lattice of non-singular vortices with diffusely distributed vorticity, this anisotropic contribution is not zero. Since the total energy of the Cooper pair is 


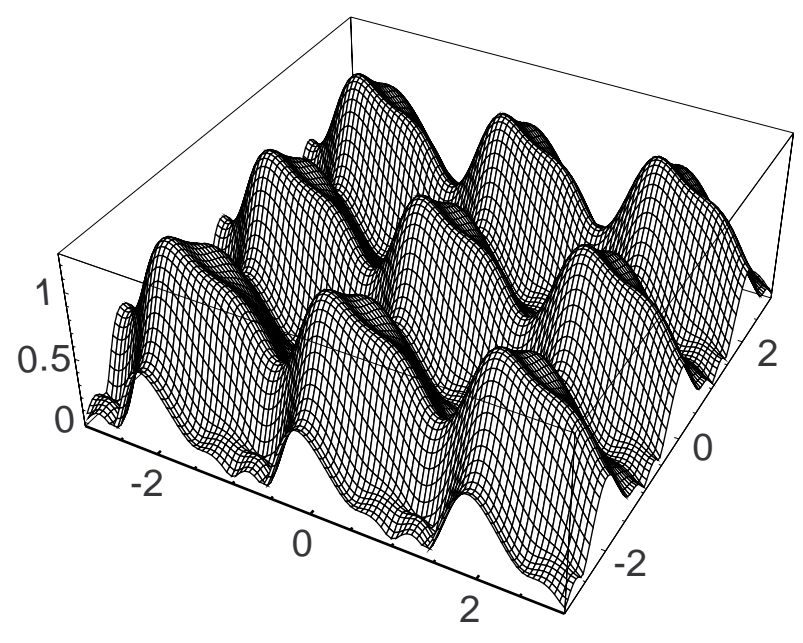

Figure 2. Distribution of the absolute value of superfluid velocity in a rotating coordinate system for a lattice of non-singular vortices with a diffusely distributed vorticity.

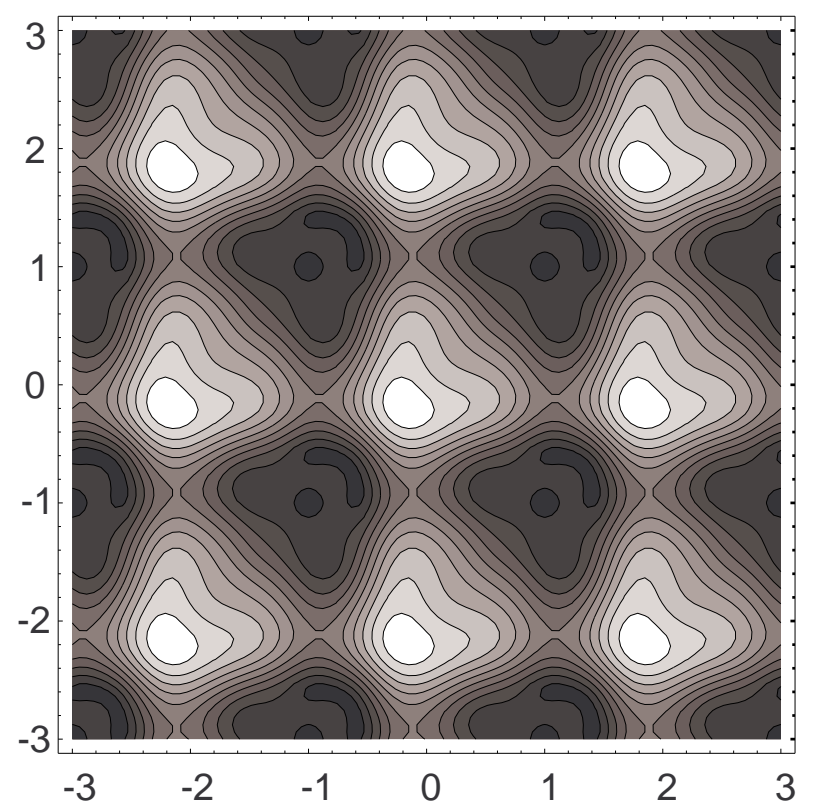

Figure 3. Contour diagram of the amplitude of superfluid velocity, allowing the reader to see the periodic lattice of continuous vortices

conserved in the vortex, its kinetic energy (and hence the velocity amplitude) is also anisotropic.

Returning to the Cartesian coordinate system $x, y, z$, we use the following simple correspondence between the complex superfluid velocity $v(x, y)$ and the three-dimensional vector $\mathbf{v}_{s}$ in a Cartesian system

$\mathbf{v}_{s}(x, y)=\frac{\hbar}{2 M a}\{\Re v(x, y), \Im v(x, y), 0\}$.

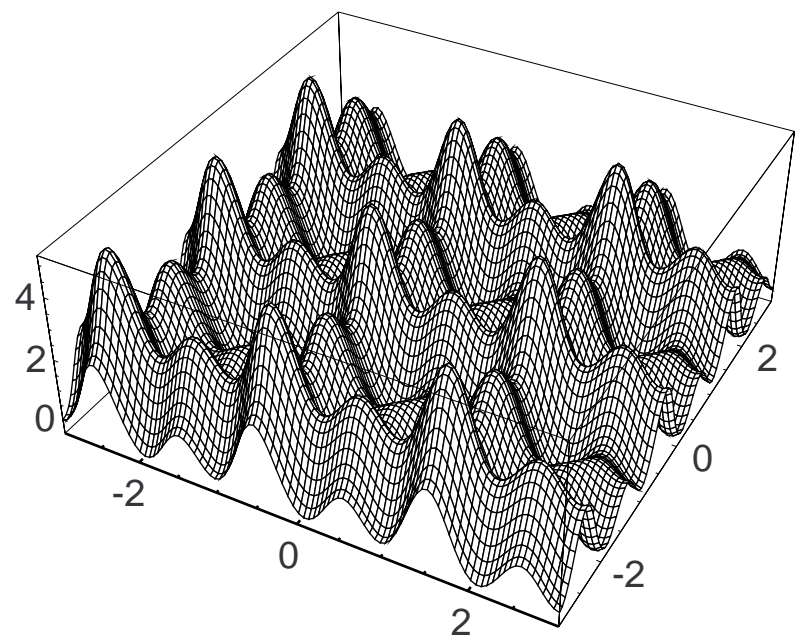

Figure 4. The diffusely distributed vorticity in the rotating ${ }^{3} \mathrm{P}_{2}$ superfluid with a lattice of non-singular vortices.

In the laboratory frame of reference, we have

$v=\frac{1}{2}(1+\cos \beta) i \zeta(x-i y+1-i)$

$+\frac{1}{2}(1-\cos \beta) i \zeta(x-i y)$.

The vorticity of the superfluid flow is diffusely distributed over the entire elementary cell of the vortex lattice $\boldsymbol{\nabla} \times \mathbf{v}_{s}=2 \frac{\hbar}{2 M a} \sin \beta(\nabla S \times \nabla \beta)$.

Since the superfluid velocity is concentrated in the plane of rotation, the vorticity vector is directed along the angular velocity $\boldsymbol{\Omega}$ and is continuously distributed over the lattice cells, as shown in Fig. 4.

In Fig. 5, we present a contour plot of this function, which allows us to see the "topographic map" of the vorticity continuously distributed over each unit cell.

Numerical integration over the cell area gives

$\int_{-a}^{a} d x \int_{-a}^{a} d y\left(\boldsymbol{\nabla} \times \mathbf{v}_{s}\right)_{z}=2 \pi$

which means that each of the cells contains a unit of the quantized vorticity $\varkappa=1$, distributed over its area. A one circulation quantum equal to $\pi \hbar / M$, therefore the vortex density must be equal to $2 M \Omega / \pi \hbar$ so that the average vorticity of the whole lattice, $\left\langle\nabla \times \mathbf{v}_{s}\right\rangle=2 \boldsymbol{\Omega}$, corresponds to a rigid rotation with an angular velocity of $\boldsymbol{\Omega}$.

\section{ESTIMATION OF FREE ENERGY}

With the function (29) we have calculated the free energy per area carrying unit vorticity:

$\mathcal{F}=\int_{\text {cell }} \tilde{F}(x, y) d x d y$

with $\tilde{F}(x, y)$ from Eq. (16), where we substitute the Cartesian superfluid velocity

$\mathbf{V}(x, y)=\{\Re \mathcal{V}(x, y), \Im \mathcal{V}(x, y), 0\}$, 


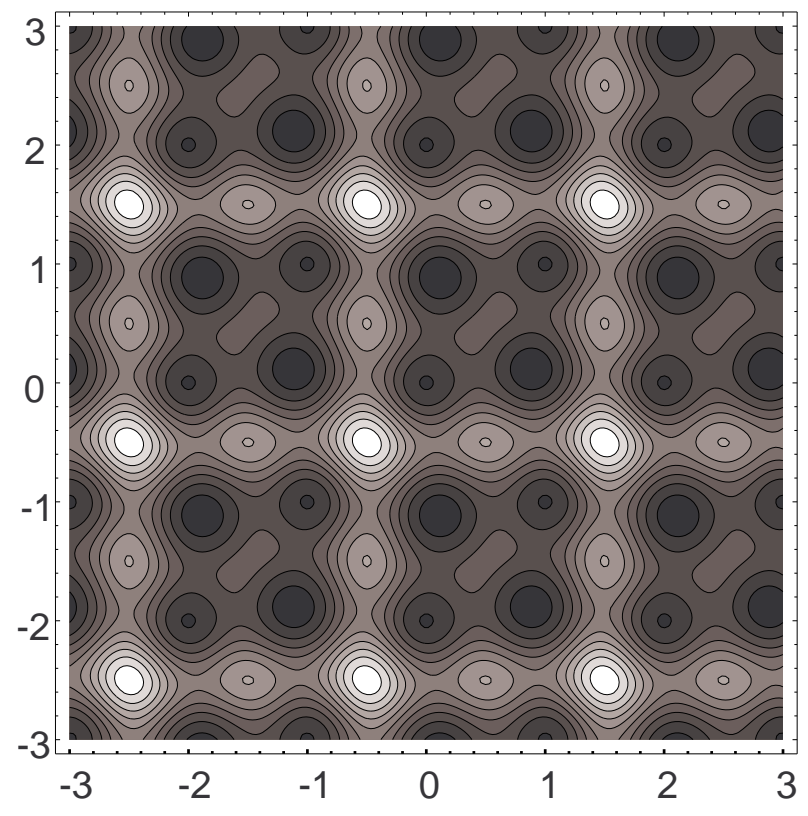

Figure 5. Contour diagram of the diffusely distributed vorticity in the rotating ${ }^{3} \mathrm{P}_{2}$ superfluid with a lattice of non-singular vortices.

with $\mathcal{V}(x, y)$ from Eq. (25) and

$\nabla S=\{\Re \Lambda(x, y), \Im \Lambda(x, y), 0\}$,

where

$\Lambda(x, y)=\frac{1}{4}[i \zeta(x-i y+1-i)-i \zeta(x-i y)]$.

Using these functions, one can numerically estimate the free energy per unit cell, as indicated in Eq. (33), to obtain $\mathcal{F}=47.7 \frac{\hbar^{2} \rho_{s}}{(2 M)^{2}}$.

Which set of vortices is the most stable for a rotating superfluid neutron system with spin-triplet pairing? To answer this question let us compare this result with the free energy of a lattice of ordinary linear singular vortices, which is believed to be the most stable in the inner core of superfluid neutron stars. As we consider the problem in the London limit, assuming spatial variations to be slow, one has to compare only derivative terms in the free energy. For the set of ordinary linear vortices the derivative part of the free energy consists of only the kinetic term since the distortion energy of the $\mathbf{l}$ field vanishes. In this case, the problem is reduced to that for the lattice of linear singular vortices in the superfluid ${ }^{4} \mathrm{He}$, which is well studied. We quote the result of Tkachenko (1965) for the energy per elementary cell of the vortex lattice carrying a unit vorticity:

$\mathcal{F}_{0}=\frac{\hbar^{2} \rho_{s}}{(2 M)^{2}}\left\{\begin{array}{l}-4.117+\pi \ln (d / \xi) \text { for the square lattice, } \\ -4.150+\pi \ln (d / \xi) \text { for the triangular lattice. }\end{array}\right.$

The number $N_{v}$ of vortices with the unit circulation created inside rotating neutron stars can be estimated to be

$N_{v} \simeq 1.9 \times 10^{19}\left(\frac{1 \mathrm{~ms}}{P}\right)\left(\frac{M^{*}}{900 \mathrm{MeV}}\right)\left(\frac{R}{10 \mathrm{~km}}\right)$,

where $P$ is the period of the neutron star, $M^{*}$ is the effective neutron mass, and $R$ is the radius of the ${ }^{3} \mathrm{P}_{2}$ superfluid. Then, we can estimate the distance between vortices $d$ from $N_{v} \pi d^{2}=\pi R^{2}$ to get

$d=2.3 \times 10^{-4}\left(\frac{900 \mathrm{MeV}}{M^{*}}\right)^{1 / 2}\left(\frac{P}{1 \mathrm{~ms}}\right)^{1 / 2}\left(\frac{R}{10 \mathrm{~km}}\right)^{1 / 2} \mathrm{~cm}$.

On the other hand, the coherence length of ${ }^{3} \mathrm{P}_{2}$ superfluid is about

$\xi \simeq \frac{\hbar v_{F}}{1.75 \pi k_{B} T_{c}} \simeq 1.1 \times 10^{-11}\left(\frac{M}{M^{*}}\right) \rho_{14}^{1 / 3}\left(\frac{10^{9} \mathrm{~K}}{T_{c}}\right) \mathrm{cm}$,

In these equations the coherence length $\xi$ for the neutrons is related to the corresponding Fermi velocity, $v_{F}$, and the superfluid-transition temperature, $T_{c}$. The neutron matter density is written in units $\rho_{14}=\rho_{n} / 10^{14} \mathrm{~g} / \mathrm{cm}^{3}$.

Since the distance $d$ between the vortices in the core of a neutron star is very large compared to the radius $\xi$ of the central filament of the vortex the logarithm in Eq. (42) is large, $\pi \ln (d / \xi) \sim 60$. This allows for the conclusion that the existence of an ordered lattice slightly changes the energy of linear singular vortices, and the single vortex approximation also gives a reasonable result. The energy per unit length of an isolated singular vortex in the spin-triplet superfluid of neutrons, as derived in (Mendell 1991) is

$\frac{E}{L}=\pi \rho_{s} \frac{\hbar^{2}}{4 M^{2}} \ln \frac{d}{\xi}$.

\section{DISCUSSION}

According to the results of the preceding section the lattice of the non-singular vortices with a vorticity diffusely distributed over the entire unit cell seems to be more energetically favourable in inner superfluid core of rotating neutron stars. This contradicts the established opinion that the motion of neutron ${ }^{3} \mathrm{P}_{2}$ superfluid in neutron stars is irrotational.

In the evaluation of the free energy we have used the trial functions and hence the value obtained is the upper bound. It is reasonable to feel that the relationship between the energies of the two structures will remain the same in the exact calculation. Of course, there appears a question to stability of the lattices we have considered. The analogous investigations on helium (Abrikosov et al. 1965) have shown that all is not well in connection with stability of lattices against long waves. The stability of the structure and the improvement of the trial functions can be further studied by the perturbation method.

If the lattice of non-singular vortices is indeed more favourable, this may affect the hydrodynamics of superfluid neutron stars. As is well known, drag and pinning effects play an important role in the current theory of pulsar glitches (Alpar et al. 1984). For recent review see e.g. (Alpar 2017). Usually the drag on neutron vortices in the core is due to 
electron scattering which depends on the magnetization of the superfluid vortices (Muzikar et al. 1980). It is easily to verify that the order parameter, defined with Eqs. (1) and (2), satisfies the unitary condition. This implies that the superfluid state under consideration retains time reversal symmetry and does not have spin polarization. In other words the core-less ${ }^{3} \mathrm{P}_{2}$ vortices have no magnetization and the electron scattering off the vortices is ineffective. By the same reason pinning with flux tubes is also impossible.

We considered vortices in neutron matter without taking into account the superfluid proton component. It can be expected that taking into account the entrainment effects can lead to magnetization of non-singular vortices. This problem deserves a separate consideration.

\section{Data availability}

The data underlying this article are available in the article and in its on-line supplementary material.

\section{REFERENCES}

Abrikosov A. A., Kemoklidze M. P., Khalatnikov I. M., 1965, Zh. Eksp. Teor. Fiz. 48, 765 [Sov. Phys. JETP 21, 506]

Alpar M. A., 2017, Journal of Astrophysics and Astronomy, 38, 44

Alpar M. A., Langer S. A., Sauls J. A., 1984, Astrophys. J. 282, 533

Chamel N., Haensel P., 2008, Living Reviews in Relativity, 11, 10

Chechetkin V. R., 1976, Zh. Eksp. Teor. Fiz. 71, 1463 [Sov. Phys. JETP 44, 766]

Feynman R. P., 1965, Progr. Low Temp. Phys. 1, 17

Fujita T., Nakahara M., Ohmi T., Tsuneto T., 1978, Prog. Theor. Phys. 60, 671

Gradshteyn I. S., Ryzhik I. M., 2007, Table of integrals, series, and products. Academic Press

Haskell B., Sedrakian A., 2018, Superfluidity and Superconductivity in Neutron Stars. pp 401-454 (arXiv:1709.10340), doi:10.1007/978-3-319-97616-7_8

Ho W. G., Espinoza C., Antonopoulou D., Andersson N., 2015, ] 10.1126/sciadv. 1500578

Kiknadze L. V., Mamaladze Y. G., Che!shvili . D., 1965, Zh. Eksp. Teor. Fiz. 48, 1520 [Sov. Phys. JETP 21, 1018]

Masuda K., Nitta M., 2016, Phys. Rev. C93, 035804

Mendell G. A., 1991, ApJ, 380, 515

Mermin N. D., Ho T. L., 1976, Phys. Rev. Lett. 36594

Mermin N. D., Stare C., 1973, Phys. Rev. Lett. 301135

Milne-Thomson L. M., 1964, Theoretical Hydrodynamics. Macmillan, London

Montoli A., Antonelli M., Pizzochero P. M., 2020, Monthly Notices of the Royal Astronomical Society, 492, 4837

Muzikar P., Sauls J. A., Serene J. W., 1980, Phys. Rev.D21, 1494

Richardson R. W., 1972, Phys. Rev. D5, 1883

Sauls J. A., Stein D. L., Serene J. W., 1982, Phys. Rev. D25, 967

Sedrakian A., Clark J. W., 2019, Eur. Phys. J. A, 55, 167

Takatsuka T., 1972, Prog. Theor. Phys. 48, 1517

Tamagaki R., 1970, Prog. Theor. Phys. 44, 905

Tkachenko V. K., 1965, Sov. Phys. JETP, 42, 164

Volovik G. E., 1984, Usp. Fiz. Nauk 14373 [Sov. Phys. Usp. 27 363]

Volovik G. E., Kopnin N. B., 1977, Pis'ma Zh. Eksp. Teor. Fiz. 25, 26 [JETP Lett. 25, 22] 